\title{
RELATIONS BETWEEN SPATIO-TEMPORAL PHENOMENA AND EIGENVALUES IN MUTUALLY COUPLED CNNS
}

\author{
Zonghuang YANG, Masayuki YAMAUCHI, Yoshifumi NISHIO and Akio USHIDA \\ Department of Electrical and Electronic Engineering, Tokushima University \\ 2-1 Minami-Josanjima, Tokushima 770-8506, JAPAN
}

\begin{abstract}
The work is concerned with investigation of spatio-temporal phenomena on mutually coupled CNN array. Besides pattern formation and active wave propagation, we found that other interesting spatio-temporal phenomena such as phaseinversion-wave and classical waves can be also observed in the same network structure. In particular, we focus our discussion on the relations of spatio-temporal phenomena and temporal eigenvalues.
\end{abstract}

\section{INTRODUCTION}

Studies of dynamic phenomena in arrays composed of chaotic [1]-[6] and oscillatory [7]-[9] elements are very important for understanding various phenomena observed in natural fields such as biology, physics, chemistry, etc. From the middle of the last decade, the investigations about the spatiotemporal dynamics in CNNs have been widely carried out, and many papers have been published, where they have discussed pattern formations and various types of autowaves such as excitability waves, concentration waves and so on. Up to the present, these CNN arrays, which are composed of chaotic oscillators [1]-[6], second order nonlinear circuit obtained by the suitably "reducing" Chua's circuit [7], and etc. have been mainly considered. A common technique in all these cases usually use the CNNs as the approximations of the various types of nonlinear partial differential equations, especial the well-known reaction-diffusion equations that show Turing pattern and propagation phenomena in various continuous media [10]. The purpose of this paper is to study spatio-temporal phenomena occurred in a simpler CNN structure - mutually coupled two-layer CNNs, based on the CNNs' temporal eigenvalues.

\section{MUTUALLY COUPLED TWO-LAYER CNNS}

The mutually coupled two-layer CNNs in this paper are described as the extension of the single-layer continuous time Chua-Yang CNN; their state equation is formulated as fol- lows:

$$
\left.\begin{array}{l}
\dot{x}_{1 ; i j}=-x_{1 ; i j}+A_{1} * y_{1 ; i j}+B_{1} * u_{1 ; i j}+C_{1} * y_{2 ; i j}+I_{1} \\
\dot{x}_{2 ; i j}=-x_{2 ; i j}+A_{2} * y_{2 ; i j}+B_{2} * u_{2 ; i j}+C_{2} * y_{1 ; i j}+I_{2}
\end{array}\right\}
$$

with output equation

$$
\left.\begin{array}{l}
y_{1, i j}=0.5\left(\left|x_{1, i j}+1\right|-\left|x_{1, i j}-1\right|\right) \\
y_{2, i j}=0.5\left(\left|x_{2, i j}+1\right|-\left|x_{2, i j}-1\right|\right)
\end{array}\right\}
$$

for $1 \leq i \leq M$ and $1 \leq j \leq N$, where two mutually coupled templates $C_{1}$ and $C_{2}$ are introduced and the subscript 1 and 2 stand for first layer and second layer of the twolayer CNN array. We assume that all cells remain in linear region, then the solution of the linear system of the CNN in general case has the following form for the simple temporal eigenvalue.

$$
\mathbf{x}(t)=\sum_{i=1}^{2 M N}\left\langle\mathbf{r}_{i}, \mathbf{x}(0)\right\rangle e^{\lambda_{i} t} \mathbf{q}_{i}
$$

where $\mathbf{x}(t)$ is the state vector, $\mathbf{q}_{i}$ is the eigenvector of the system matrix, $\mathbf{r}_{i}$ represents the reciprocal basis, $\mathbf{x}(0)$ is the initial condition, and $\langle.,$.$\rangle denotes the scalar product in$ $\mathbf{R}^{2 M N}$. The solution shows various possibilities for the different ranges of the temporal eigenvalues. In the following section, we investigate the spatio-temporal phenomena based on the temporal eigenvalues in the two-layer CNNs.

\section{SPATIO-TEMPORAL PHENOMENA}

\subsection{Pattern Formations and Autowaves}

Many nonlinear partial differential equations (PDEs) have shown to generate various types of nonlinear spatio-temporal phenomena. The concept of reaction-diffusion CNNs have been formalized to reproduce similar phenomena in [1], because they are mathematically described by a discretized version of the following well-known system of nonlinear partial equations - reaction-diffusion equations:

$$
\frac{\partial \boldsymbol{u}}{\partial t}=\boldsymbol{f}(\boldsymbol{u})+\boldsymbol{D} \nabla^{2} \boldsymbol{u}
$$




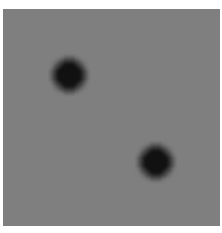

(a)

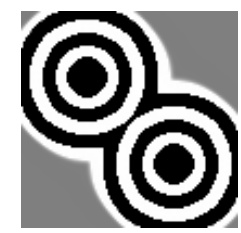

(b)

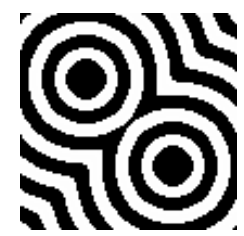

(c)
Figure 1: An example of tripe pattern formation with $D_{1}=$ 0.2 . (a) is initial state, (b) is transient result, and (c) is steady output result.

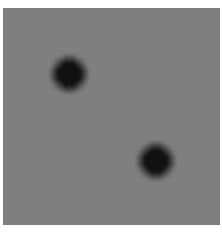

(a)

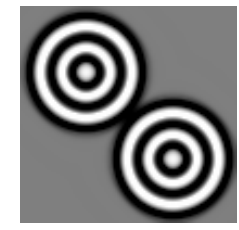

(b)

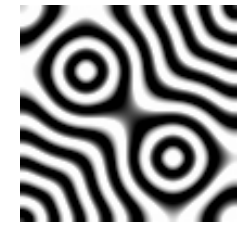

(c)
Figure 2: An example of active wave propagation phenomena. (a) is initial state, (b) is transient result, and (c) is steady output result.

where $\boldsymbol{D}$ is a diagonal matrix, which describes the intensity of diffusive coupling between the components of $\boldsymbol{u}$. Similarly, if we use the two-layer CNNs to approximate the above reaction-diffusion equation with $\boldsymbol{u} \in \mathbf{R}^{2}, \boldsymbol{f} \in \mathbf{R}^{2}$, then the two-layer CNNs can be written as follows:

$$
\left.\begin{array}{l}
\dot{x}_{1, i j}=-x_{1, i j}+a_{1} y_{1, i j}+c_{1} y_{2, i j}+d_{1} \nabla^{2} y_{1, i j} \\
\dot{x}_{2, i j}=-x_{2, i j}+a_{2} y_{2, i j}+c_{2} y_{1, i j}+d_{2} \nabla^{2} y_{2, i j}
\end{array}\right\}
$$

Thus, We have the following results that, if at least one of the temporal eigenvalues has positive real part, the CNN constructed by the connection between diffusing cells will become unstable in the linear region, and behave as pattern formations or active propagation phenomena. The necessary condition is described by (6), which is directly derived from those conditions obtained Turing patterns in array of coupled circuits [7].

$$
\left.\begin{array}{l}
\frac{\left(a_{1}-1\right)}{d_{1}}+\frac{\left(a_{2}-1\right)}{d_{2}}>0 \\
{\left[\left(a_{1}-1\right) d_{2}-\left(a_{2}-1\right) d_{1}\right]^{2}>-4 d_{1} d_{2} c_{1} c_{2}}
\end{array}\right\}
$$

Figure 1 shows a simulation example for pattern formation, which is carried out under a two-layer $\mathrm{CNN}$ array consisted of $100 \times 100$ cells with zero-flux boundary condition and the following parameter set: $a_{1}=2.6, a_{2}=2.6$, $d_{1}=0.1, d_{2}=0.01, c_{1}=-1$, and $c_{2}=1$. In Fig. 1, (a), (b) and (c) are respectively the initial state, transient result and steady output of the CNN. The simulation result indicates that, the stripe pattern formation process starts from the objects in the image (i.e. non-zero initial conditions), propagates toward around the objects, generates new stripes with almost the same geometry with the shape of original initiated objects. Then, it propagates again until the whole CNN array reaches a steady state. While the propagation wave front collides with another wave front or the boundary, the wave fronts annihilate without any interference and reflection. Moreover, the density of stripe pattern can be adjusted by suitably changing the template parameters. From this example, we have seen that the shape of stripe pattern is dependent on its initial state of the CNN array. Thus, many interesting stripe patterns such as the coats of animal, fingerprint, and etc. may be regenerated by suitably selecting initial conditions of the CNN array and the CNN parameters.

Figure 2 shows another simulation example for active propagation phenomena, which is carried out under the same conditions with the last example. This simulation indicates that two concentric circular waves are generated. Their wave fronts propagate in all directions through the network from the initialized positions. After the waves fronts collide with boundary or collide with each other, they annihilate without any interference and reflection. This propagation phenomenon behaves as autowaves do.

\subsection{Phase Wave Propagation Phenomena}

A lot of studies on phase relationship of coupled oscillators or chaotic circuits have been widely carried out [6][9]. One of interesting spatio-temporal phenomena - phase inversion wave has been observed in van der Pol oscillators coupled by inductors as a ladder [9]. In this section, we will investigate similarly dynamic behaviors encountered in the simpler two-layer CNN array. Obviously, the CNN must satisfy the following two conditions that the nonlinear second-order cell in absence of coupling to its neighbor cells oscillates with a limit cycle, and the whole CNN is unstable (i.e., at least one of the temporal eigenvalues has positive real). Next, let us observe how the phase difference between adjacent cells propagates the $\mathrm{CNN}$ array. In Fig. 3(b), the vertical axes are the sum of the first layer outputs of adjacent cells, the horizontal axes are time. Hence, the diagram shows qualitatively how phase differences between adjacent cells change as time goes, where the white regions correspond to the state that two adjacent cell/oscillators are anti-phase synchronization, and the black regions to the inphase synchronization. Figure 3(b) shows an example for the wave propagation phenomena of the phase difference that is observed from one-dimensional two-layer $\mathrm{CNN}$ array composed of 18 cells with zero-flux boundary condition. The initial state condition of the first layer of this CNN array is set as Fig. 3(a), where the black squares denote having value 1 and the white squares value -1 , and the initial state condition of the second layer is zero. The template is

$$
A_{1}=\left[\begin{array}{lll}
-0.03 & 1.1 & -0.03
\end{array}\right], C_{1}=\left[\begin{array}{lll}
0 & -3 & 0
\end{array}\right] \text {, }
$$




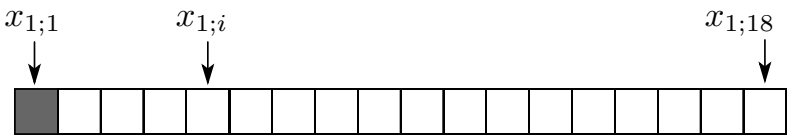

(a)

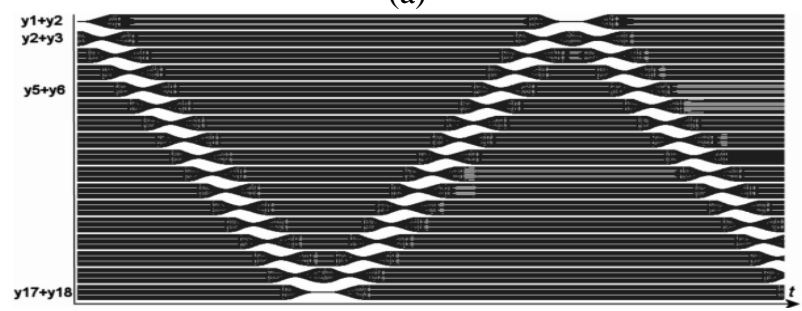

(b)

Figure 3: Phase wave propagation phenomenon in onedimensional CNN array of 18 cells.

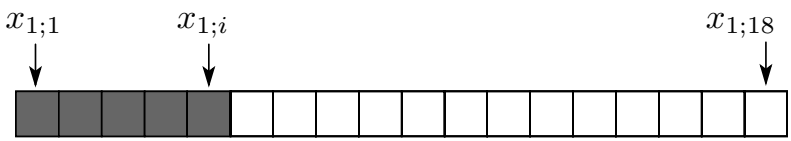

(a)

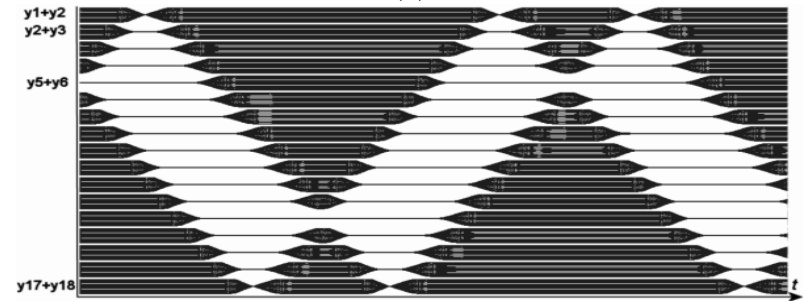

(b)

Figure 4: Anothor phase wave propagation phenomenon in one-dimensional CNN array with different initial condition.

$$
\begin{array}{r}
A_{2}=\left[\begin{array}{lll}
0.03 & 1.1 & 0.03
\end{array}\right], C_{2}=\left[\begin{array}{lll}
0 & 5 & 0
\end{array}\right], \\
B_{1}=B_{2}=0, I_{1}=I 2=0 .
\end{array}
$$

Figure 4(b) shows another example for phase wave propagation with the different initial state conditions shown by Fig. 4(a). We observe that the anti-phase between adjacent cells is transferred gradually from one end to the other end of the CNN array, and is reflected when the states of anti-phase collide with the end of array, or collide with each other. This propagation process of phase exists continuously in the two-layer CNN array, so called "phase-inversion-wave" [9]. The two simulations indicate that the phase wave propagation phenomena can be reproduced in the two-layer CNN with simpler structure.

\subsection{Classical Wave Propagation}

We have shown in the above subsections that the two-layer CNNs can reproduce many interesting nonlinear phenomena, in which there is a common property that at least one of temporal eigenvalues of the $\mathrm{CNNs}$ has positive real part. However, when all of the temporal eigenvalues have zero or negative real parts, what happens in the CNNs? We consider the phenomena in this subsection.

Let us consider an autonomous two-layer CNN with mutual coupling between layers. Its equation is described as the following form:

$$
\left.\begin{array}{rl}
\dot{x}_{1, i j}= & -x_{1, i j}+\left(a_{1}+1\right) y_{1, i j}+c_{1} y_{2, i j} \\
& +d_{1} \nabla^{2} y_{2, i j} \\
\dot{x}_{2, i j}= & -x_{2, i j}+\left(a_{2}+1\right) y_{2, i j}+c_{2} y_{1, i j} \\
& +d_{2} \nabla^{2} y_{1, i j} .
\end{array}\right\}
$$

We assume that all cells remain in the linear region $\left(y_{1, i j}=\right.$ $x_{1, i j}, y_{2, i j}=x_{2, i j}$ ). Thus, the equation (8) can be solved by decoupling method [9], and its solution can be expressed as a weighted sum of $M \times N$ orthogonal space dependent eigenfunctions $\phi_{M N}(m, n ; i, j)$ in the following form:

$\left.\begin{array}{l}x_{1, i j}(t)=\sum_{m=0}^{M-1 N-1} \sum_{\substack{m=0 \\ M-1 N-1}}\left(\alpha_{m n} e^{\lambda_{m n 1}} \beta_{m n} e^{\lambda_{m n 2} t}\right) \phi_{M N}(m, n ; i, j) \\ \left.x_{2, i j}(t)=\sum_{m=0} \sum_{n=0}^{\lambda_{m n} e^{\lambda_{m n 1}}} \delta_{m n} e^{\lambda_{m n 2} t}\right) \phi_{M N}(m, n ; i, j)\end{array}\right\}$

where $\alpha_{m n}, \beta_{m n}, \gamma_{m n}, \delta_{m n}$ are constants depending on the initial conditions. $\lambda_{m n 1}, \lambda_{m n 2}$ are temporal eigenvalues, which are influenced by spatial eigenvalue $k_{m n}^{2}$ corresponding to spatial eigenfunction.

$$
\begin{aligned}
\lambda_{m n}\left[k_{m n}^{2}\right] & =\frac{1}{2}\left[\left(a_{1}+a_{2}\right) \pm\right. \\
& \left.\quad \sqrt{\left(a_{1}-a_{2}\right)^{2}+4\left(k_{m n}^{2} d_{2}-c_{2}\right)\left(k_{m n}^{2} d_{1}-c_{1}\right)}\right]
\end{aligned}
$$

For the zero-flux boundary condition, the spatial eigenfunction and eigenvalue can be assumed as the following form:

$$
\nabla^{2} \phi_{M N}(m, n ; i, j)=\cos \frac{(2 i+1) m \pi}{2 M} \cos \frac{(2 j+1) n \pi}{2 N}
$$

and

$$
k_{m n}^{2}=4\left(\sin ^{2} \frac{m \pi}{2 M}+\sin ^{2} \frac{n \pi}{2 N}\right)
$$

The equation (9) is important because the cell states of the two-layer CNN are given as the time-dependent weighted sums of spatial eigenfunctions. From this equation, it can be derived that, if all the temporal eigenvalues have zero or negative real part, all the states of the $\mathrm{CNN}$ are in the linear region. Thus, the CNNs have the properties of the linear space, such as superposition etc. Therefore, we can use this $\mathrm{CNN}$ to model passive media with/without loss in the linear space.

Figure 5 shows a simulation example for the wave propagation phenomenon in a two-layer CNN array composed of $100 \times 100$ cells, which is executed by selecting the following parameter set: $a_{1}=0, a_{2}=0, d_{1}=-0.5, d_{2}=0.5$, 


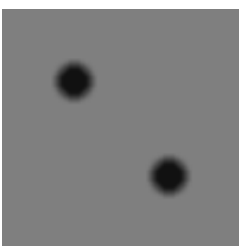

(a)

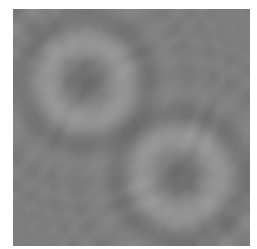

(b)

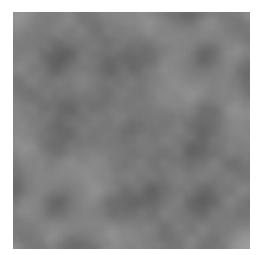

(c)
Figure 5: An example for the passive wave propagation phenomena without loss. (a) is initial state, (b) and (c) show two snapshots observed in different times.

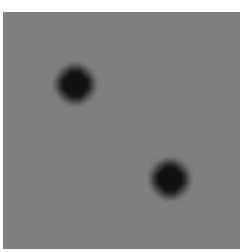

(a)

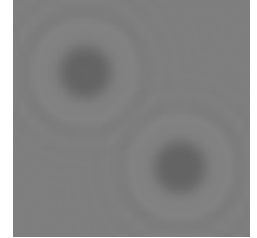

(b)

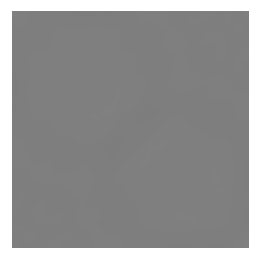

(c)
Figure 6: An example for the passive wave propagation phenomena with loss. (a) is initial state, (b) and (c) show two snapshots observed in different times.

$c_{1}=-5$, and $c_{2}=5$ (i.e., all the temporal eigenvalues of the $\mathrm{CNN}$ are complex numbers with zero real part and the isolated cell behaves as oscillator with limited cycle). The simulation result shows that the circular waves propagating from the initialized position are generated. These circular waves propagate in all directions through the plane array and their amplitudes decrease with propagating. When the waves collide with each other, they don't annihilate and reflect, but permeate through and superpose on each other. When they collide with the boundary, the waves are reflected. Moreover, after the waves are repeatedly reflected and superposed, the initialized positions become invisible. These behaviors are completely different form those of nonlinear propagation phenomena, but behave as the classical wave does. In this case, the CNN can be used for modeling passive media without loss. On the other hand, if we select the parameter set to satisfy all temporal eigenvalues of the CNN having negative real parts, then each cell state will decay to zero, which can be derived from Eq. (9). This situation is suitable for modeling passive media with loss. For example, if the parameter set: $a_{1}=-0.04, a_{2}=0$, $d_{1}=-0.5, d_{2}=0.5, c_{1}=-5$, and $c_{2}=5$ is selected, then all the temporal eigenvalues of the CNN are complex numbers with negative real part and the isolated cell without couple behaves as damped oscillator. Figure 6 shows the simulation result. From the analysis of the simulation result, we can see that the propagation phenomenon has the same properties with the above one. However, the network is calm at the end due to the temporal eigenvalues having negative real part.

\section{CONCLUSIONS}

In this paper, we have investigated the spatio-temporal phenomena in the two-layer CNNs in term of the temporal eigenvalues, and found many interesting spatio-temporal phenomena. The necessary conditions for these phenomena have been indicated. Based on our research results, the two-layer CNN can be considered not only for modeling active media, but also for modeling passive media with/without loss. Moreover, we found that an interesting phase wave propagation phenomena can be produced in the same two-layer CNN structure. All of these facts show that the two-layer CNNs have a real potential for applications.

\section{REFERENCES}

[1] L. O. Chua, M. Hasler, G. S. Mochytz, and J. Neirynck, "Autonomous cellular neural networks: a unified paradigm for pattern formation and active wave propagation," IEEE Trans. Circuits \& Syst., vol.42, no.10, pp.559-577, 1995.

[2] V. P. Munuzuri, V. P. Villar, and L. O. Chua, "Autowaves for image processing on a two-dimensional CNN array of excitable nonlinear circuits: flat and wrinkled labyrinths," IEEE Trans. Circuits \& Syst., vol.40, no.3, pp.174-181, 1995.

[3] M.J. Ogorzalek, Z. Galias, A.M. Dabrowski, and W.R. Dabrowki, "Chaotic waves and spatio-temporal patterns in large arrays of doubly-coupled Chua's circuits," IEEE Trans. Circuits \& Syst., vol.42, no.10, pp.706-714, 1995.

[4] V. I. Nekorkin, V. B. Kazantsev, and L. O. Chua, "Chaotic attractors and waves in a one-dimensional array of modified Chua's circuits," Int. J. Bifurcation and Chaos, vol.6, no.7, pp.1295-1317, 1995.

[5] V. L. Zheleznyak and L. O. Chua, "Coexistence of low- and high-dimensional spatiotemporal chaos in a chain of dissipatively coupled Chua's circuit," Int. J. Bifurcation and Chaos, vol.4, no.3, pp.639-674, 1994.

[6] Y. Nishio and A. Ushida, "Spatio-temporal chaos in simple coupled chaotic circuits," IEEE Trans. Circuits \& Syst., vol.42, no.10, pp.678-686, 1995.

[7] L. Goras, L.O. Chua, D.M.W. Leenaerts, and L. Pivka, "Turing patterns in CNNs part I-III," IEEE Trans. Circuits \& Syst., vol.42, no.10, pp.602-637, Oct. 1995.

[8] G. Manganaro, P. Arena and L. Fortuna, "Cellular neural networks,” Springer-Verlag, Berlin, Heidelberg, 1999.

[9] M. Yamauchi, M. Wada, Y. Nishio, and A. Ushida, "Wave propagation phenomena of phase states in oscillators coupled by inductor as a ladder," IEICE Trans. Fundamentals, vol.E82-A, no.11, pp.2592-2598, 1999.

[10] J. D. Murray, Mathematical biology, Springer, Berlin, 1989. 